\title{
Risk Factors for Thyroid Dysfunction among Type 2 Diabetic Patients in a Highly Diabetes Mellitus Prevalent Society
}

\author{
Metab Al-Geffari, ${ }^{1}$ Najlaa A. Ahmad, ${ }^{2}$ Ahmad H. Al-Sharqawi, ${ }^{2}$ Amira M. Youssef, ${ }^{3}$ \\ Dhekra AlNaqeb, ${ }^{4}$ and Khalid Al-Rubeaan ${ }^{5}$ \\ ${ }^{1}$ Family and Community Medicine Department, Qassim University, P.O. Box 143, Buraidah 51411, Saudi Arabia \\ ${ }^{2}$ Biostatistics Department, University Diabetes Center, King Saud University, P.O. Box 245, Riyadh 11411, Saudi Arabia \\ ${ }^{3}$ Registry Department, University Diabetes Center, King Saud University, P.O. Box 245, Riyadh 11411, Saudi Arabia \\ ${ }^{4}$ Research Department, University Diabetes Center, King Saud University, P.O. Box 245, Riyadh 11411, Saudi Arabia \\ ${ }^{5}$ University Diabetes Center, King Saud University, P.O. Box 18397, Riyadh 11415, Saudi Arabia
}

Correspondence should be addressed to Khalid Al-Rubeaan; krubean@ksu.edu.sa

Received 3 October 2013; Accepted 1 December 2013

Academic Editor: Jack R. Wall

Copyright (C) 2013 Metab Al-Geffari et al. This is an open access article distributed under the Creative Commons Attribution License, which permits unrestricted use, distribution, and reproduction in any medium, provided the original work is properly cited.

Diabetes and thyroid dysfunction found to exist simultaneously. In this regard, the present study looked into the prevalence of different forms of thyroid dysfunction and their risk factors among Type 2 diabetic Saudi patients. Methodology. A crosssectional retrospective randomized hospital-based study of 411 Type 2 diabetic Saudi patients of $>25$ years of age was conducted to test the prevalence of different types of thyroid dysfunction and their risk factors. Results. The prevalence of different types of thyroid dysfunction is $28.5 \%$, of which $25.3 \%$ had hypothyroidism, where $15.3 \%, 9.5 \%$, and $0.5 \%$ are clinical, subclinical, and overt hypothyroidism, respectively. The prevalence of hyperthyroidism is $3.2 \%$, of which subclinical cases accounted for $2.7 \%$ and overt hyperthyroidism accounted for $0.5 \%$. Risk factors for thyroid dysfunction among Saudi Type 2 diabetic patients are family history of thyroid disease, female gender, and duration of diabetes of $>10$ years, while the risk was not significant in patients with history of goiter and patients aged $>60$ years. Smoking and parity show a nonsignificant reduced risk. Conclusion. Thyroid dysfunction is highly prevalent among Saudi Type 2 diabetic patients, and the most significant risk factors are family history of thyroid disease, female gender, and $>10$ years duration of diabetes.

\section{Introduction}

Diabetes mellitus and thyroid dysfunction are the most common endocrine diseases seen in the adult population [1], while insulin or thyroid hormones metabolism can result in functional abnormalities of one another. The strong link between diabetes and thyroid diseases encouraged the American Diabetes Association (ADA) to propose that people with diabetes must be checked periodically for thyroid dysfunction [2]. Thyroid disease should be screened annually in diabetic patients to detect asymptomatic thyroid dysfunction [3]. At the same time, patients with thyroid dysfunction may need to be tested for the possibility of abnormal glucose metabolism, since excessive thyroid hormones cause increased glucose production in the liver, rapid absorption of glucose through the intestine, and increased insulin resistance [4]. The thyroid gland is one of the endocrinal systems of the human body and can be affected by sustained hyperglycemia and the continuous endeavors by the body to correct for this carbohydrate imbalance. Studies have shown that diabetes and thyroid dysfunction can be found to exist together where thyroid disease can affect glucose metabolism and the untreated thyroid dysfunction can affect the management of diabetes $[3,5]$. The association of the two endocrinal dysfunctions has been reported in different societies throughout the last two decades [6-8]. Diabetic patients have susceptibility to different types of thyroid dysfunction, whether hypothyroidism or hyperthyroidism; at the same time, patients with thyroid dysfunction are susceptible to suffer from either Type 1 diabetes or Type 2 diabetes $[1,9]$. 
Thyroid disorder is divided into clinical and subclinical disease, according to the hormonal levels and clinical presentation that will affect the follow-up and management plan. Thyroid dysfunction has been found to be more prevalent among diabetic population when compared with the normal population [5].

In Scotland, the prevalence of thyroid dysfunction was $13.4 \%$ among diabetics, reaching $31.4 \%$ in Type 1 female diabetic patients and falling to $6.9 \%$ in Type 2 male diabetic patients [6], while among Type 2 diabetic patients in Jordan, the overall prevalence of thyroid dysfunction was found to be $12.5 \%$ [8]. Subclinical hypothyroidism prevalence is variable among different ethnic groups or genders and was found to range from 4.8 to $6.3 \%[6,10]$. This was clearly shown in the United States, where prevalence was $5.8 \%$ in white women and $1.2 \%$ in black women but $3.4 \%$ in white men and $1.8 \%$ in black men [11].

Hyperthyroidism is a less common thyroid dysfunction in both general and diabetic patients. It has been reported to be $0.53 \%$ in Caucasian children with Type 1 diabetes mellitus [12] and $4.4 \%$ in Type 2 diabetic adult patients [13], while subclinical hyperthyroidism is reported to be approximately $2 \%[14]$.

There are many risk factors known to be associated with thyroid dysfunction in the general population, including age, gender, BMI, family history of thyroid disease, smoking, and pregnancy. Incidence of hyperthyroidism and hypothyroidism increases with age, especially beyond 20 years, and it has been established that female gender is $10-20$ times more likely to have this medical problem than males [15]. Morbidly obese individuals show a high prevalence of overt and subclinical hypothyroidism, accounting for 19.5\% [16]. The United Kingdom DNA collection for Graves' disease and Hashimoto's thyroiditis study identified family history of thyroid disease to be risk for thyroid dysfunction [17].

Smoking has been reported to be a risk for thyroid dysfunction, where higher T4 levels and lower TSH levels were reported among smokers but not among nonsmokers or former smokers. This may be explained by the toxicological effect of smoking on increasing levels of thyroxin binding globulin among smokers [18].

Estrogen has been shown to be associated with low risk for thyroid dysfunction, while pregnancy has a higher risk for developing hyperthyroidism [19].

Risk factors for thyroid dysfunction among diabetic patients are similar to what have been reported in nondiabetics, although they will vary with the type of thyroid dysfunction. Autoimmune thyroid disease is seen to be more frequent in the younger age group and females [20], while hypothyroidism among diabetic patients is more prevalent among women [21] and the older population [22]. Diabetes duration has been found to be a risk for thyroid autoimmune disuses in children and adolescents with type 1 diabetes [23], but it was not a risk in patients suffering from Type 2 diabetes in different ethnic groups $[10,24]$. Goiter has been recognized as a risk factor for thyroid dysfunction in diabetic patients [10], as observed in nondiabetics [25]. Parity has been recognized to be a risk factor for thyroid dysfunction in diabetic women [26], which is also the case in nondiabetic mothers [27].

Saudi Arabia is the seventh of the top ten countries in terms of the prevalence of diabetes among the adult population aged 20-79, according to the IDF diabetes atlas 2012 [28]. The prevalence of thyroid dysfunction among Saudi diabetic patients was reported to be $16 \%$, as opposed to $7 \%$ in nondiabetics, as shown by Akbar et al. in 2006 [7]. Since then, no study has been undertaken to investigate the relationship between diabetes and thyroid dysfunction, or to examine their risk factors in a community with high diabetes prevalence.

Since most studies investigating the prevalence of thyroid disease in diabetic patients have focused on Type 1 diabetes, the aim of this study is to assess the prevalence of different forms of thyroid dysfunction among Type 2 diabetic Saudi patients receiving care from April to October in 2012 at the University Diabetes Center (UDC) in King Abdul Aziz University Hospital (KAUH) in Riyadh. Determining the risk factors of thyroid dysfunction among Type 2 diabetic Saudi patients is part of this study's objectives.

\section{Methodology}

This study is a cross-sectional retrospective randomized hospital-based study, in which 411 Type 2 diabetic Saudi patients were enrolled during the period April to October in 2012. The UDC is a tertiary diabetes center, which provides care for diabetic patients in Riyadh, the capital of Saudi Arabia.

Subjects recruited for this study were Saudi nationals with Type 2 diabetes of more than 25 years of age. The diagnosis of Type 2 diabetes was based on their initial presentation, using the American Diabetes Association (ADA) Criteria [29]. Inclusion criteria included adult Saudi Type 2 diabetic patients older than 25 years visiting the UDC during the study period. Exclusion criteria included patients who had previous thyroid surgery, pregnant women, Type 1 diabetes mellitus, and patients on the following medications: cordarone "antiarrhythmic medication" lithium, interferon, iodide, or high doses of glucocorticoids.

Chart review was conducted to collect data, including demographic parameters that is, age, gender, and duration of diabetes, in addition to anthropometric measurements including weight, height, and body mass index (BMI) in addition to blood pressure that was collected from their last visit. Family history of diabetes or thyroid disease with or without goiter was reported, in addition to smoking history and parity for females. The presence of any associated diseases like hypertension, dyslipidemia, and thyroid disease including goiter was also documented. Laboratory data were collected from the patients' chart of the last visit, including HbAlc, fasting blood sugar (FBG), and 2 hour postprandial (2hpp) glucose, in addition to lipids profile including total cholesterol, triglyceride, high density lipoprotein (HDL), and low density lipoprotein (LDL). Thyroid function tests, namely, thyroid-stimulating hormone (TSH), free thyroxine 
(FT4) and free thyroxine (FT3), were collected during the same visit.

Each patient is evaluated for the presence of thyroid dysfunction, defined as biochemical abnormalities for clinical and subclinical hypothyroidism and hyperthyroidism, if they had been diagnosed and treated with either hypothyroidism or hyperthyroidism. Patients were classified as having clinical hypothyroidism if they have been diagnosed before and on thyroxin replacement therapy. Patients were labeled with sub-clinical hypothyroidism if they have TSH $>5 \mathrm{mIU} / \mathrm{L}$ but normal T4 (10.55-25.74 pmol/L), while overt hypothyroidism when TSH $>5.0 \mathrm{mIU} / \mathrm{L}$ with low T4 $<10.55 \mathrm{pmol} / \mathrm{L}$. Patients were labeled with hyperthyroidism if they have been treated surgically or given radioactive iodine therapy or on antithyroid medications. Diagnosis of subclinical hyperthyroidism is when TSH $<0.5 \mathrm{mIU} / \mathrm{L}$ with normal T4 (10.55$25.74 \mathrm{pmol} / \mathrm{L}$ ), while overt hyperthyroidism is when TSH $<$ $0.5 \mathrm{mIU} / \mathrm{L}$ with high T4 $>25.74 \mathrm{pmol} / \mathrm{L}[30,31]$.

\section{Statistical Analysis}

Data were entered into SPSS software version 17.0. Continuous variables were expressed as mean \pm standard deviation, and categorical variables were expressed, as percentages. $t$ test was used for continuous variables and chi square test for categorical variables. Relative risk with $95 \%$ confidence interval (CI) was used to assess different risk factors of thyroid dysfunction among Type 2 diabetic patients. $P$ value of less than 0.05 was used as a level of significance, and GraphPad software was used to plot different relative risk factors.

\section{Results}

Thyroid dysfunctions were found in 117 patients (28.5\%) of the total sample of 411 Type 2 DM Saudi patients. The patients' baseline characteristics of the total sample showed a mean age of $59.0 \pm 10.8$ years but $59.3 \pm 9.9$ and $58.9 \pm 11.3$ for diabetic subjects with and without thyroid dysfunction, respectively, which is not significantly different $(P=0.732)$. Female gender percentage in the total sample was $52.3 \%$ but was significantly higher in patients with thyroid dysfunction of $68.6 \%$ when compared with normal thyroid subjects, where females accounted for $46.6 \%$ with $P$ value $<0.0001$. The mean diabetes duration was also significantly higher in patients with thyroid dysfunction than in normal ones $(17.3 \pm 9.0$ versus $15.1 \pm 8.6$, resp.) with $P$ value $=0.032$. The percentage of patients with a positive family history of thyroid disease was significantly higher in patients with thyroid dysfunction (14.7\%) versus (1.03\%) among the normal thyroid diabetic patients with $P$ value $<0.0001$, while the percentage of family history of diabetes was not statistically different between the two groups. The percentage of smoking habits in the two groups did not show any significant difference.

The mean weight is $80.1 \pm 15.4$ and BMI of $31.5 \pm 6.1$ for the total sample, but without a significant difference between the patients with or without thyroid dysfunction. The height is significantly lower in patients with thyroid dysfunction, compared with the normal thyroid patients $(157.3 \pm 8.2$

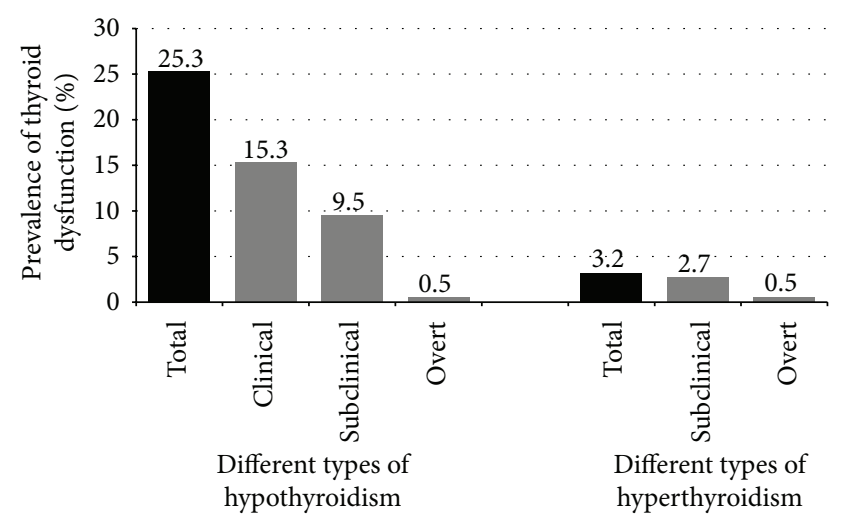

Figure 1: Prevalence of different types of thyroid dysfunction among Type 2 diabetic Saudi patients. Clinical hypothyroidism for patients receiving thyroxin treatment, and Subclinical hypothyroidism when $\mathrm{TSH}>5.0 \mathrm{mIU} / \mathrm{L}$ with normal T4 (10.55-25.74 pmol/L), while overt hypothyroidism when TSH $>5.0 \mathrm{mIU} / \mathrm{L}$ with low T4 $<10.55 \mathrm{pmol} / \mathrm{L}$. Subclinical hyperthyroidism when TSH $<0.5 \mathrm{mIU} / \mathrm{L}$ with normal T4 (10.55-25.74 pmol/L) and overt hyperthyroidism when TSH < $0.5 \mathrm{mIU} / \mathrm{L}$ with high T4 $>25.74 \mathrm{pmol} / \mathrm{L}$.

versus $161.2 \pm 9.5$, resp.) with $P$ value $<0.0001$. The mean systolic and diastolic blood pressures for the selected patients are $134.8 \pm 16.5$ and $74.1 \pm 9.6$, respectively, but did not show significant difference in patients with or without thyroid dysfunction. Goiter was found in $2.8 \%$ of the studied samples and in $4.8 \%$ in patients with thyroid dysfunction but $2.1 \%$ in patients without thyroid dysfunction, although it was not significant. The mean HbAlc, FBS, triglyceride, total cholesterol, HDL, and LDL for total sample were $8.5 \pm$ $1.6,8.7 \pm 2.9,1.5 \pm 0.78,4.2 \pm 0.78,1.2 \pm 0.33$, and $2.3 \pm$ 0.69 , respectively, but there was no significant difference for patients with or without thyroid dysfunction.

Thyroid function tests showed a significantly higher mean TSH value for patients with thyroid dysfunction than normal ones $(4.7 \pm 4.2$ versus $2.6 \pm 1.2$, resp.; $P$ value $<0.0001)$ and FT4 (16.6 \pm 4.34 versus $15.6 \pm 2.7$ with $P$ value 0.048$)$, but no significant difference for FT3 $(4.5 \pm 2.03$ versus $4.7 \pm 0.84$, resp., with $P$ value 0.552 ) as shown in Table 1 .

Figure 1 shows the prevalence of different types of thyroid dysfunction among the studied population, where the total prevalence of hypothyroidism was $25.3 \%$ and $3.2 \%$ for hyperthyroidism. The prevalence of different types of hypothyroidism includes clinical cases (15.3\%), sub-clinical (9.5\%), and overt hypothyroidism ( $0.5 \%)$. The prevalence of sub-clinical hyperthyroidism in the studied sample was $2.7 \%$ and $0.5 \%$ for overt hyperthyroidism.

The relative risk and 95\% CI for different risk factors for thyroid dysfunction, namely, hypo- or hyperthyroidism for Saudi Type 2 diabetic patients, are shown in Figure 2. Positive family history for thyroid diseases the strongest risk factor with a relative risk (RR) of 3.39 (95\% CI, 2.47-4.63) with $P$ value $<0.0001$, followed by female gender with RR of 1.95 (95\% CI, 1.36-2.78) with $P$ value $<0.0001$. A duration of diabetes of more than 10 years has a relative risk of 1.66 (95\% CI, 1.06-2.61) and $P$ value $=0.019$. Other risk factors, 
TABLE 1: Baseline characteristics of study sample for all subjects with or without thyroid dysfunction among Type 2 diabetic patients aged $>25$ years.

\begin{tabular}{|c|c|c|c|c|}
\hline & $\begin{array}{c}\text { All samples } \\
n=411\end{array}$ & $\begin{array}{c}\text { Diabetic subjects with } \\
\text { thyroid dysfunction } \\
n=117\end{array}$ & $\begin{array}{l}\text { Diabetic subjects without } \\
\text { thyroid dysfunction } \\
n=294 \\
\end{array}$ & $P$ value \\
\hline Age (years) ${ }^{*}$ & $59.0 \pm 10.8$ & $59.3 \pm 9.9$ & $58.9 \pm 11.3$ & 0.732 \\
\hline Female gender ${ }^{\dagger}$ & 52.3 & 68.6 & 46.6 & $<0.0001$ \\
\hline Duration of diabetes in years ${ }^{*}$ & $15.8 \pm 8.6$ & $17.3 \pm 9.0$ & $15.1 \pm 8.6$ & 0.032 \\
\hline Family history of diabetes ${ }^{\dagger}$ & 86.3 & 84.2 & 87.1 & 0.487 \\
\hline Family history of thyroid disease $^{\dagger}$ & 5.4 & 14.7 & 1.03 & $<0.0001$ \\
\hline Positive smoking history ${ }^{\dagger}$ & 17.4 & 14.3 & 18.2 & 0.516 \\
\hline Weight in kilogram $(\mathrm{Kg})^{*}$ & $80.1 \pm 15.4$ & $78.6 \pm 15.9$ & $80.6 \pm 15.2$ & 0.268 \\
\hline Height $(\mathrm{cm})^{*}$ & $159.9 \pm 9.2$ & $157.3 \pm 8.2$ & $161.2 \pm 9.5$ & $<0.0001$ \\
\hline $\operatorname{BMI}\left(\mathrm{kg} / \mathrm{m}^{2}\right)^{*}$ & $31.5 \pm 6.1$ & $32.0 \pm 6.2$ & $31.2 \pm 5.8$ & 0.209 \\
\hline Systolic blood pressure $(\mathrm{mmHg})^{*}$ & $134.8 \pm 16.5$ & $134.6 \pm 16.9$ & $134.5 \pm 16.2$ & 0.926 \\
\hline Diastolic blood pressure $(\mathrm{mmHg})^{*}$ & $74.1 \pm 9.6$ & $73.1 \pm 8.3$ & $74.4 \pm 10.0$ & 0.198 \\
\hline Presence of goiter ${ }^{\dagger}$ & 2.8 & 4.8 & 2.1 & 0.248 \\
\hline $\operatorname{HbAlc}(\%)^{*}$ & $8.5 \pm 1.6$ & $8.5 \pm 1.5$ & $8.5 \pm 1.6$ & 0.943 \\
\hline Fasting blood sugar $(\mathrm{mmol} / \mathrm{L})^{*}$ & $8.7 \pm 2.9$ & $8.6 \pm 3.0$ & $8.6 \pm 2.8$ & 0.904 \\
\hline Triglycerides $(\mathrm{mmol} / \mathrm{L})^{*}$ & $1.5 \pm 0.78$ & $1.7 \pm 0.80$ & $1.5 \pm 0.82$ & 0.110 \\
\hline Total cholesterol $(\mathrm{mmol} / \mathrm{L})^{*}$ & $4.2 \pm 0.78$ & $4.3 \pm 0.96$ & $4.2 \pm 0.68$ & 0.429 \\
\hline $\mathrm{HDL}(\mathrm{mmol} / \mathrm{L})^{*}$ & $1.2 \pm 0.33$ & $1.3 \pm 0.38$ & $1.2 \pm 0.31$ & 0.190 \\
\hline $\mathrm{LDL}(\mathrm{mmol} / \mathrm{L})^{*}$ & $2.3 \pm 0.69$ & $2.2 \pm 0.81$ & $2.3 \pm 0.62$ & 0.427 \\
\hline FT3 $(\mathrm{pmol} / \mathrm{L})^{*}$ & $4.6 \pm 1.4$ & $4.5 \pm 2.03$ & $4.7 \pm 0.84$ & 0.552 \\
\hline $\mathrm{FT} 4(\mathrm{pmol} / \mathrm{L})^{*}$ & $15.9 \pm 3.5$ & $16.6 \pm 4.34$ & $15.6 \pm 2.7$ & 0.048 \\
\hline $\mathrm{TSH}(\mathrm{mIU} / \mathrm{L})^{*}$ & $3.4 \pm 2.9$ & $4.7 \pm 4.2$ & $2.6 \pm 1.2$ & $<0.0001$ \\
\hline
\end{tabular}

${ }^{*}$ Data presented as mean $\pm \mathrm{SD},{ }^{\dagger}$ Data presented as (\%), and $P$ value is calculated between diabetic subjects with and without thyroid dysfunction.

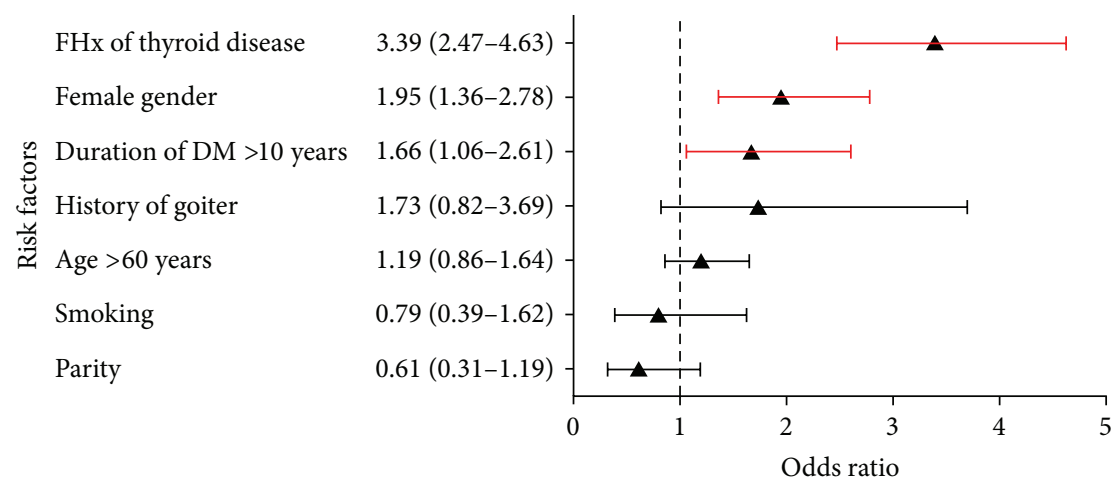

(a)

\begin{tabular}{lcc}
\hline Risk factor & Relative risk and 95\% CI & $P$-value \\
\hline Family history of thyroid disease & $3.39(2.47-4.63)$ & $<0.0001$ \\
Female gender & $1.95(1.36-2.78)$ & $<0.0001$ \\
Duration of diabetes $\geq 10$ years & $1.66(1.06-2.61)$ & 0.019 \\
History of goiter & $1.73(0.82-3.69)$ & 0.248 \\
Age $>60$ years & $1.19(0.86-1.64)$ & 0.296 \\
Smoking & $0.79(0.39-1.62)$ & 0.516 \\
Parity & $0.61(0.31-1.19)$ & 0.251 \\
\hline
\end{tabular}

(b)

FIGURE 2: Relative risks for thyroid dysfunction among Type 2 Saudi diabetic patients; (a) forest plot, (b) table of values. 
namely, history of goiter and age of more than 60 years, have a relative risk of 1.73 with $95 \%$ CI (0.82-3.69) and 1.19 with $95 \%$ CI (0.86-1.64) respectively, but both were nonsignificant, with $P$ value $=0.248$ and 0.296 , respectively. Smoking and parity showed low nonsignificant relative risk of 0.79 (0.39$1.62)$, and $0.61(0.31-1.19)$ with $P$ value $=0.516$ and $=0.251$, respectively.

\section{Discussion}

This study has demonstrated that thyroid dysfunction affects more than one quarter of Saudi Type 2 diabetic patients, which is more than that has been reported by Akbar et al. of $16 \%$ in 2006 [7] although this study has a bigger sample and older cohort age. In this study, we report the highest prevalence of thyroid dysfunction in Type 2 diabetic patients when compared with other communities, shown by the Scotland study to be $13.4 \%$ among both Type 1 diatebes and Type 2 diabetes [6] or by the Jordanian study, where it was $12.5 \%$ among Type 2 diabetes [8]. This could be explained by the high prevalence of latent autoimmune diabetes of adult (LDA) in Saudi Type 2 diabetics reaching $26 \%$ [7] and in the current study, those patients are not excluded. When comparing our findings of different types of thyroid dysfunction for similar Type 2 diabetic patients' cohort from Spain published by Díez et al. in 2011 [10], we had identical findings for the prevalence of both total and subclinical hypothyroidism cases $(25.3 \%$ versus $25.8 \%$ and $9.5 \%$ versus $10.7 \%$ resp.). The prevalence of clinical cases of hypothyroidism was $15.3 \%$ in our subjects, while overt hypothyroidism in Spain was $15.1 \%$. We had a lower prevalence of total hyperthyroid cases when compared with Spain study $(3.2 \%$ versus $6.6 \%)$ but not for subjects with subclinical hyperthyroidism. Overt hyperthyroid cases were higher in the Spain study when compared with our study (3.5\% versus $0.5 \%)$.

Diabetic patients with a positive family history of thyroid disease had a higher chance of developing thyroid dysfunction, while the family history of diabetes did not increase the risk for thyroid dysfunction which is the same observation in the United Kingdom DNA collection for Graves' disease and Hashimoto's thyroiditis study [17]. Among Saudi Type 2 diabetic patients of more than 25 years of age, positive family history of thyroid disease is the most prominent risk factor for thyroid dysfunction, as is also shown in Caucasians [32].

As shown in many ethnic groups $[8,20]$, Saudi diabetic patients with thyroid dysfunction had a significant predominance of female gender. Diabetes duration of more than 10 years in our cohort has been shown to be an important risk factor, which is not the case in the studies of different ethnic groups like Spanish and Chinese population [10, 24]. This may explained by the fact that our cohort had a marked longer duration of 7.3 years versus 9.6 years in Spanish and 8.3 years in Chinese.

We did not find history of goiter to be a significant risk factor for thyroid dysfunction in this retrospective study which was similar to what has been reported by Díez et al. in a more representative prospective study [10], although history of goiter is recognized as a risk factor for thyroid dysfunction in general population [33]. Our results have denied that age is a significant risk factor for thyroid dysfunction, as with what has been reported by different studies $[10,34]$. While smoking has been identified as a risk factor for thyroid dysfunction in the general population, especially when smoking is highly prevalent [35], no studies have investigated the impact of smoking as a risk among the diabetic population. We report here that smoking has no effect on thyroid dysfunction among Type 2 diabetic patients. This finding in Type 2 diabetic patients is reported for the first time and has to be taken with special caution, since smoking has very low prevalence among Saudi females for cultural reasons [36].

Parity has been reported in many studies to be a risk factor for thyroid dysfunction in the general population $[19,26]$, but we did not find this to be the case among Saudi Type 2 diabetic females, although diabetic pregnant women have an increased risk of developing postpartum thyroiditis [27].

Although thyroid autoimmunity is strong risk factors for thyroid dysfunction among diabetic patients, the current retrospective study lacks thyroid antibody data.

We conclude that more than one quarter of Saudi Type 2 diabetic patients of more than 25 years of age are affected by different types of thyroid dysfunction, whereby the majority are hypothyroid cases. Our findings regarding the prevalence of different thyroid dysfunction among Type 2 Saudi diabetic population are higher than those that have been reported by most of studies conducted in different ethnic groups. We have found that family history of thyroid disease, female gender, and duration of diabetes for more than 10 years are significant risk factors for different thyroid dysfunctions.

Based on a high prevalence of thyroid dysfunction among Saudi Type 2 diabetic patients, routine screening for thyroid dysfunction is highly recommended in Saudi diabetic population.

\section{Acknowledgments}

The study was approved by the Institutional Review Board (IRB) of the College of Medicine Research Center (CMRC). The authors would like to acknowledge the great support of research staff in research unit at the University Diabetes Center for their support in conducting that study.

\section{References}

[1] L. H. Duntas, J. Orgiazzi, and G. Brabant, "The interface between thyroid and diabetes mellitus," Clinical Endocrinology, vol. 75, no. 1, pp. 1-9, 2011.

[2] American Diabetes Association, "Standards of medical care in diabetes-2013," Diabetes Care, vol. 36, no. 1, pp. S11-S66, 2013.

[3] R. S. Gray, W. J. Irvine, and B. F. Clarke, "Screening for thyroid dysfunction in diabetics," British Medical Journal, vol. 2, no. 6202, p. 1439, 1979.

[4] J. L. Johnson, "Diabetes control in thyroid disease," Diabetes Spectrum, vol. 19, no. 3, pp. 148-153, 2006.

[5] J. Feely and T. E. Isles, "Screening for thyroid dysfunction in diabetics," British Medical Journal, vol. 1, no. 6179, p. 1678, 1979. 
[6] P. Perros, R. J. McCrimmon, G. Shaw, and B. M. Frier, "Frequency of thyroid dysfunction in diabetic patients: value of annual screening," Diabetic Medicine, vol. 12, no. 7, pp. 622-627, 1995.

[7] D. H. Akbar, M. M. Ahmed, and J. Al-Mughales, "Thyroid dysfunction and thyroid autoimmunity in Saudi type 2 diabetics," Acta Diabetologica, vol. 43, no. 1, pp. 14-18, 2006.

[8] A.-R. M. Radaideh, M. K. Nusier, F. L. Amari et al., "Thyroid dysfunction in patients with type 2 diabetes mellitus in Jordan," Saudi Medical Journal, vol. 25, no. 8, pp. 1046-1050, 2004.

[9] R. Kadiyala, R. Peter, and O. E. Okosieme, "Thyroid dysfunction in patients with diabetes: clinical implications and screening strategies," International Journal of Clinical Practice, vol. 64, no. 8, pp. 1130-1139, 2010.

[10] J. J. Díez, P. Sánchez, and P. Iglesias, "Prevalence of thyroid dysfunction in patients with type 2 diabetes," Experimental and Clinical Endocrinology and Diabetes, vol. 119, no. 4, pp. 201-207, 2011.

[11] J. G. Hollowell, N. W. Staehling, W. Dana Flanders et al., "Serum TSH, T4, and thyroid antibodies in the United States population (1988 to 1994): National Health and Nutrition Examination Survey (NHANES III)," Journal of Clinical Endocrinology and Metabolism, vol. 87, no. 2, pp. 489-499, 2002.

[12] F. Lombardo, M. F. Messina, G. Salzano et al., "Prevalence, presentation and clinical evolution of graves' disease in children and adolescents with type 1 diabetes mellitus," Hormone Research in Paediatrics, vol. 76, no. 4, pp. 221-225, 2011.

[13] M. F. Celani, M. E. Bonati, and N. Stucci, "Prevalence of abnormal thyrotropin concentrations measured by a sensitive assay in patients with type 2 diabetes mellitus," Diabetes Research, vol. 27, no. 1, pp. 15-25, 1994.

[14] C. Wang, "The relationship between type 2 diabetes mellitus and related thyroid diseases," Journal of Diabetes Research, vol. 2013, Article ID 390534, 9 pages, 2013.

[15] R. W. V. Flynn, T. M. MacDonald, A. D. Morris, R. T. Jung, and G. P. Leese, "The thyroid epidemiology, audit, and research study: thyroid dysfunction in the general population," Journal of Clinical Endocrinology and Metabolism, vol. 89, no. 8, pp. 38793884, 2004.

[16] M. A. Michalaki, A. G. Vagenakis, A. S. Leonardou et al., "Thyroid function in humans with morbid obesity," Thyroid, vol. 16, no. 1, pp. 73-78, 2006.

[17] K. Boelaert, P. R. Newby, M. J. Simmonds et al., "Prevalence and relative risk of other autoimmune diseases in subjects with autoimmune thyroid disease," American Journal of Medicine, vol. 123, no. 2, pp. 183-el, 2010.

[18] C. L. Fisher, D. M. Mannino, W. H. Herman, and H. Frumkin, "Cigarette smoking and thyroid hormone levels in males," International Journal of Epidemiology, vol. 26, no. 5, pp. 972977, 1997.

[19] T. G. A. Strieder, M. F. Prummel, J. G. P. Tijssen, E. Endert, and W. M. Wiersinga, "Risk factors for and prevalence of thyroid disorders in a cross-sectional study among healthy female relatives of patients with autoimmune thyroid disease," Clinical Endocrinology, vol. 59, no. 3, pp. 396-401, 2003.

[20] R. W. Holl, B. Böhm, U. Loos, M. Grabert, E. Heinze, and J. Homoki, "Thyroid autoimmunity in children and adolescents with type 1 diabetes mellitus. Effect of age, gender and HLA type," Hormone Research, vol. 52, no. 3, pp. 113-118, 1999.

[21] G. E. Umpierrez, K. A. Latif, M. B. Murphy et al., "Thyroid dysfunction in patients with type 1 diabetes: a longitudinal study," Diabetes Care, vol. 26, no. 4, pp. 1181-1185, 2003.
[22] S. A. P. Chubb, W. A. Davis, and T. M. E. Davis, "Interactions among thyroid function, insulin sensitivity, and serum lipid concentrations: the Fremantle diabetes study," Journal of Clinical Endocrinology and Metabolism, vol. 90, no. 9, pp. 5317-5320, 2005.

[23] O. Kordonouri, A. Klinghammer, E. B. Lang, A. GrütersKieslich, M. Grabert, and R. W. Holl, "Thyroid autoimmunity in children and adolescents with type 1 diabetes: a multicenter survey," Diabetes Care, vol. 25, no. 8, pp. 1346-1350, 2002.

[24] J.-K. Yang, W. Liu, J. Shi, and Y.-B. Li, "An association between subclinical hypothyroidism and sight-threatening diabetic retinopathy in type 2 diabetic patients," Diabetes Care, vol. 33, no. 5, pp. 1018-1020, 2010.

[25] M. P. J. Vanderpump, W. M. G. Tunbridge, J. M. French et al., "The incidence of thyroid disorders in the community: a twenty-year follow-up of the Whickham Survey," Clinical Endocrinology, vol. 43, no. 1, pp. 55-68, 1995.

[26] N. Friedrich, S. Schwarz, J. Thonack, U. John, H. Wallaschofski, and H. Völzke, "Association between parity and autoimmune thyroiditis in a general female population," Autoimmunity, vol. 41, no. 2, pp. 174-180, 2008.

[27] F. Santini, L. Chiovato, R. Rocchi, M. Marinò, and A. Pinchera, "Influences of thyroid diseases in diabetic pregnant women," Annali dell'Istituto Superiore di Sanita, vol. 33, no. 3, pp. 441445, 1997.

[28] "IDF Diabetes Atlas 5th edition 2012 update," International Diabetes Federation, http://www.idf.org/diabetesatlas/5e/theglobal-burden.

[29] American diabetes Association, "Standards of Medical care in diabetes-2010," Diabetes Care, vol. 33, supplement 1, pp. S11S61, 2010.

[30] G. Caldwell, H. A. Kellett, and S. M. Gow, "A new strategy for thyroid function testing," The Lancet, vol. 1, no. 8438, pp. 11171119, 1985.

[31] H. Lindner, "The Non-Clinical ATA/AACE 2012 Hypothyroidism Guidelines," http://hormonerestoration.com/files/ ResponsetoGuidelines.pdf.

[32] Z. Schroner, I. Lazurova, and J. Petrovicova, "Autoimmune thyroid diseases in patients with diabetes mellitus," Bratislavské Lekárske Listy, vol. 109, no. 3, pp. 125-129, 2008.

[33] A. Aminorroaya, M. Janghorbani, M. Amini, S. Hovsepian, A. Tabatabaei, and Z. Fallah, "The prevalence of thyroid dysfunction in an iodine-sufficient area in Iran," Archives of Iranian Medicine, vol. 12, no. 3, pp. 262-270, 2009.

[34] S. Hajieh, M. Behbahani, and A. Z. Mohtashami, "Prevalence of thyroid dysfunction and thyroid auto antibodies in type 2 diabetic patients," Pakistan Journal of Medical Sciences, vol. 27, no. 5, pp. 1169-1172, 2011.

[35] P. Vestergaard, L. Rejnmark, J. Weeke et al., "Smoking as a risk factor for Graves' disease, toxic nodular goiter, and autoimmune hypothyroidism," Thyroid, vol. 12, no. 1, pp. 69-75, 2002.

[36] J. S. Jarallah, K. A. Al-Rubeaan, A. R. A. Al-Nuaim, A. A. AlRuhaily, and K. A. Kalantan, "Prevalence and determinants of smoking in three regions of Saudi Arabia," Tobacco Control, vol. 8, no. 1, pp. 53-56, 1999. 


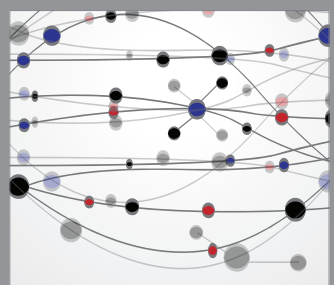

The Scientific World Journal
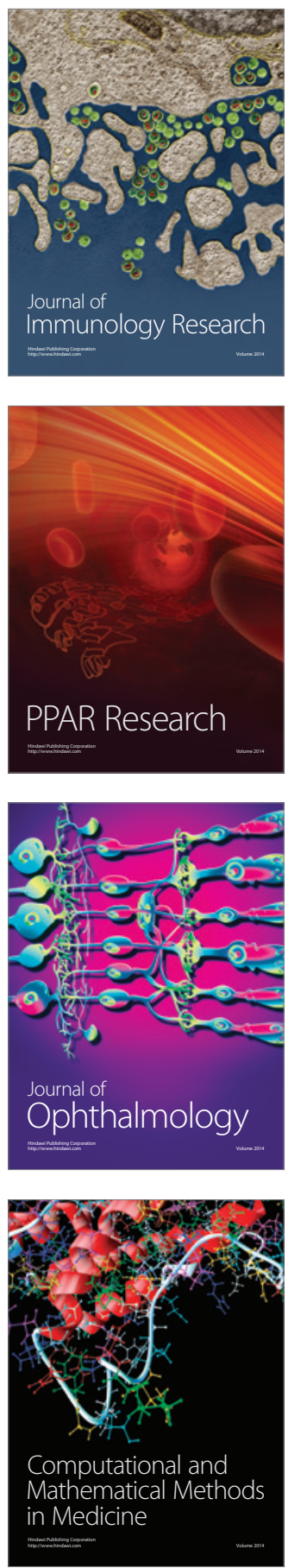

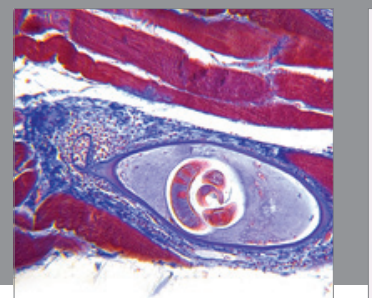

Gastroenterology

Research and Practice
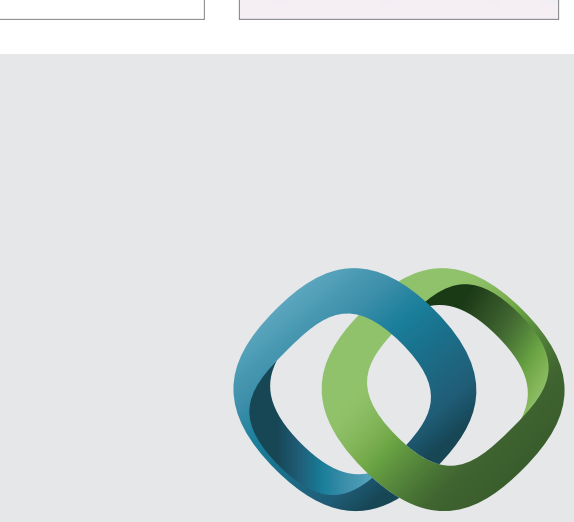

\section{Hindawi}

Submit your manuscripts at

http://www.hindawi.com
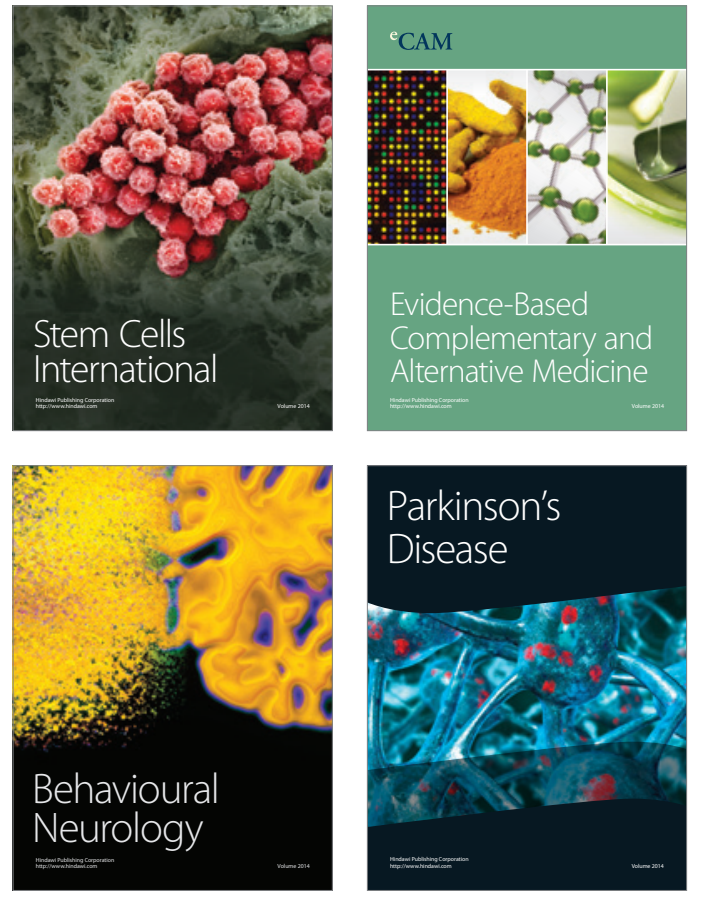
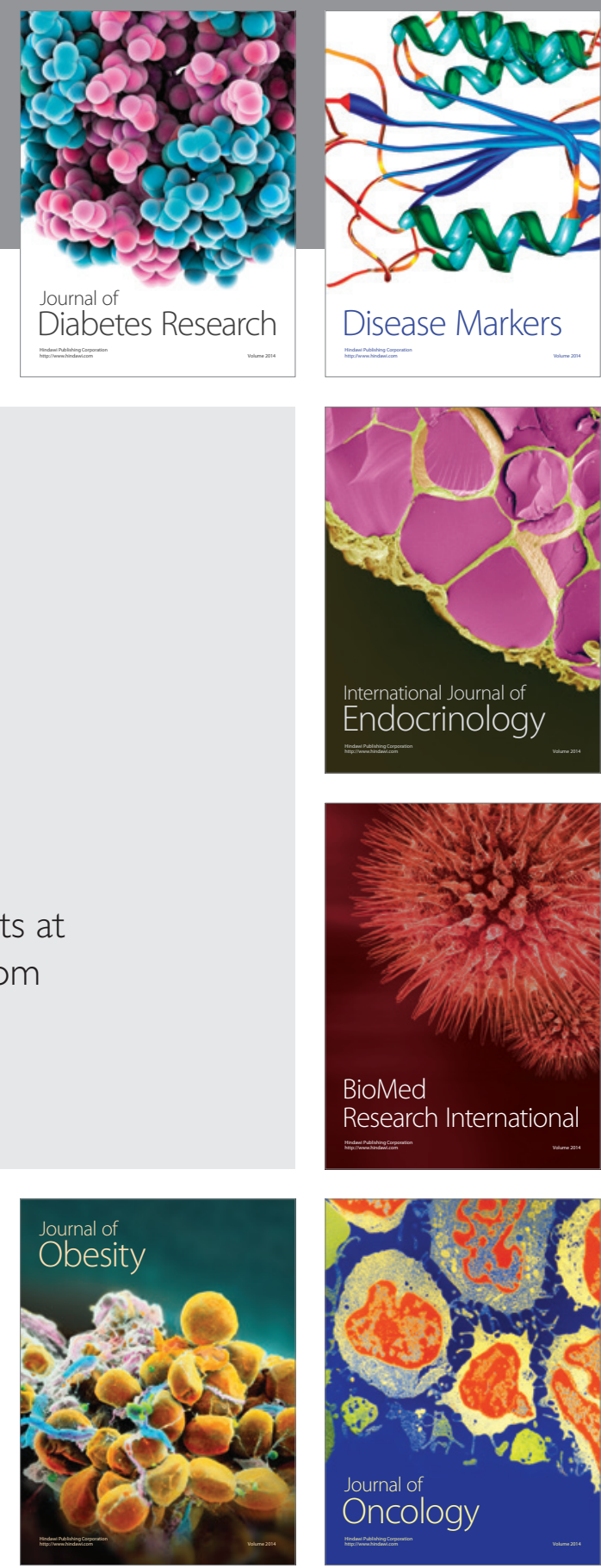

Disease Markers
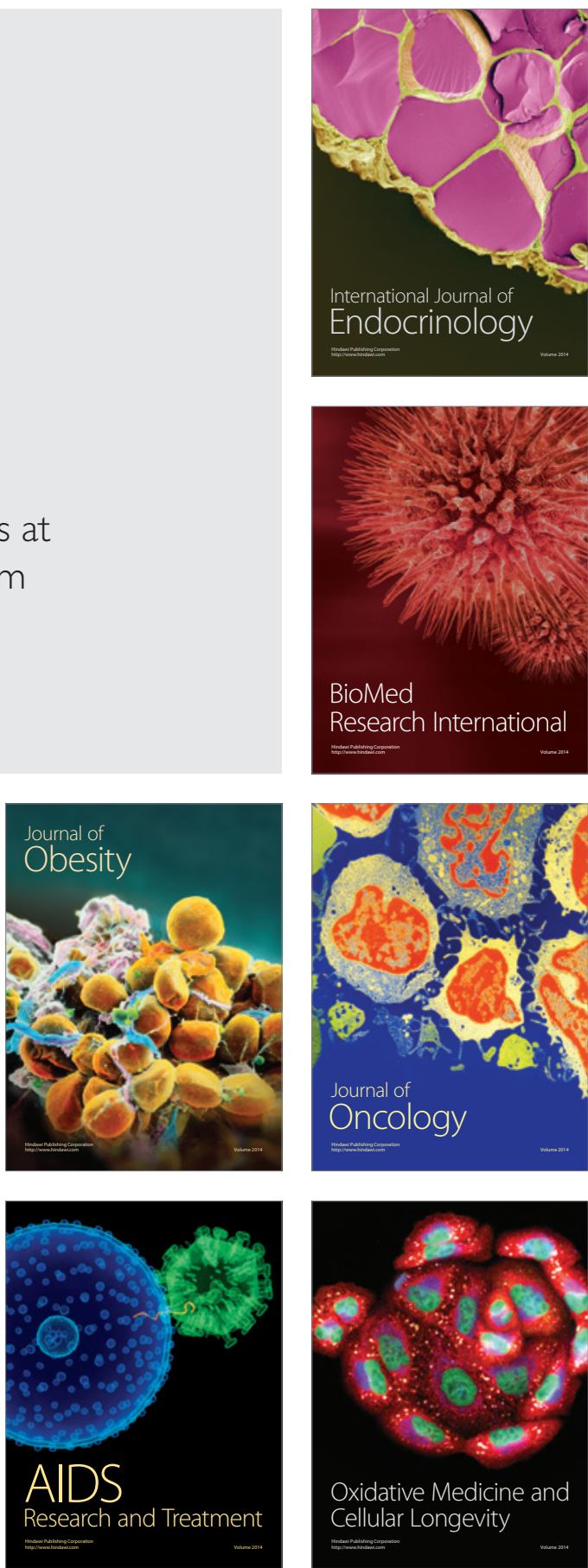